\title{
Worauf kommt es an? Qualitätssicherung im agilen Lernen
}

\author{
Joana Jungclaus
}

Das agile Sprintlernen wurde im Rahmen des Vorhabens inMEDIASres (gefördert mit Mitteln des Bundesministeriums für Bildung und Forschung und des Europäischen Sozialfonds ESF) - als eine Form des agilen Lernens im Unternehmen - von den Forschungspartnern Fraunhofer-Institut für Arbeitswirtschaft und Organisation IAO und ZNL Transfer Zentrum für Neurowissenschaften und Lernen entwickelt und gemeinsam mit vier Unternehmen zu jeweils mehreren Lernthemen erprobt.

Diese Praxisumsetzungen wurden im Rahmen des Forschungsvorhabens eng begleitet und evaluiert. Die Beobachtungen zeigen, dass mittels agilem Lernen im Unternehmen auch komplexe Lerninhalte bei verschiedensten Zielgruppen erfolgreich gelernt werden können - allerdings nur, wenn dabei einige Aspekte für die qualitativ hochwertige Ausgestaltung des agilen Lernens beachtet werden. Auch die Zufriedenheit der Lernenden und die Akzeptanz der Lernform werden dadurch beeinflusst, wie gut das agile Lernprojekt aufgesetzt und durchgeführt wurde. Dieses Kapitel beschreibt qualitätsrelevante Aspekte im agilen Lernen ausgehend von den Erfahrungen und Evaluationsergebnissen im Vorhaben inMEDIASres.

\subsection{Qualität im agilen Lernen}

Agiles Lernen im Unternehmen lässt sich für verschiedene Lerninhalte und Zielgruppen anpassen, wie die unterschiedlichen Fallbeispiele aufzeigen. Es ist kein ,fertig evaluiertes Bildungsangebot“, vielmehr ein Rahmenkonzept, das für jede Umsetzung im Unterneh-

J. Jungclaus $(\bowtie)$

ZNL TransferZentrum für Neurowissenschaften und Lernen, Ulm, Deutschland

E-Mail: joana.jungclaus@znl-ulm.de 
men inhaltlich angepasst und erneut ausgestaltet wird. Verglichen mit einem klassischen Seminar oder Workshop hat es eine deutlich komplexere Rollenstruktur und jeder Funktionsträger hat eine Reihe von Aufgaben - und damit einen Einfluss auf die Umsetzungsqualität des agilen Lernens. Letztlich spielen auch betriebswirtschaftliche Aspekte eine Rolle: Durch das agile Lernen sollen nicht nur erfolgreich neue Kompetenzen erworben werden, dies muss auch mit einem angemessenen Aufwand-Nutzen-Verhältnis für das Unternehmen und die Lernenden erfolgen.

Unter einer qualitativ hochwertigen Umsetzung von agilem Lernen verstehen wir daher folgendes:

- Das Kernziel - der Erwerb von Handlungskompetenzen - wird erreicht. Das heißt auch, dass alle Lernziele, die für das Lernthema relevant sind, erreicht werden.

- Alle Beteiligten bewerten die Lernform agiles Lernen im Unternehmen positiv und sind zufrieden mit den Ergebnissen.

- Das Lernen erfolgt bedarfsgerecht, nachhaltig und flexibel, es wird stetig angepasst an die durch die Arbeit vorgegebenen Strukturen und Rahmenbedingungen.

- Das Verhältnis von Aufwand und Nutzen ist für das Unternehmen wie für die Lernenden mindestens zufriedenstellend.

\subsection{Qualitätskriterien für das agile Lernen}

Abhängig vom Lerngegenstand stellen sich schon vor Beginn des agilen Lernens verschiedene Fragen: Wer ist der fachliche Begleiter für dieses Lernthema? Welche Lernziele werden verfolgt und wie können diese gut durch die Lernaufgaben und Akzeptanzkriterien abgedeckt werden? Wie lang sind die Etappen und lernt das Team arbeitsintegriert oder in Vollzeit (im Detail siehe auch Kap. 11)?

Das Lernen selbst geschieht nah am oder gar integriert in den Arbeitsalltag und ist durch ein kontinuierliches Zusammenspiel der Lernenden untereinander sowie mit den fachlichen und methodischen Begleitern gekennzeichnet. Die Erfahrungen aus den Erprobungen zeigen, dass hier auch Haltungsfragen ins Spiel kommen. Worauf es im Einzelnen ankommt, damit die oben genannten Qualitätsziele erreicht werden können, wird im Folgenden entlang der einzelnen Phasen und Rollen im agilen Lernen dargestellt.

\section{Vorbereitungsphase}

Zunächst ist wichtig, dass agiles Lernen nur für geeignete Lernthemen zum Einsatz kommt. Das erste Qualitätskriterium ist deshalb, dass die Eignung des Lernthemas für agiles Lernen explizit geprüft wurde. Agiles Lernen eignet sich dann, wenn Wissen und Fähigkeiten in komplexen oder mehrdeutigen Situationen zur Anwendung kommen müssen, wenn Mitarbeiter Hintergründe und Zusammenhänge erkennen müssen, wenn sie eigene Entscheidungen treffen und/oder Problemlösungsansätze entwickeln müssen. 
Neben der Eignung ist auch die Relevanz des Lernthemas entscheidend - und zwar aus Sicht des Unternehmens und aus Sicht der Lernenden. Gerade wegen der hohen Eigenaktivität und Selbststeuerung im Lernen ist es wichtig, dass das Lernthema für den eigenen Arbeitskontext relevant und sinnstiftend ist.

In dieser frühen Phase der Vorbereitung ist außerdem entscheidend, dass die Lernziele und Erwartungen des Auftraggebers transparent und vollständig geklärt werden. Andernfalls können sich im späteren Verlauf Zielkonflikte ergeben. Damit einher gehen auch eine transparente Aufwandsschätzung und die Freigabe der benötigten Ressourcen insbesondere für die zeitlichen Kapazitäten der Lernenden und der Begleiter - durch den Auftraggeber. Wenn Erwartungen nicht transparent und/oder Zeitbudgets nicht offiziell freigegeben sind, ergeben sich fast zwangsläufig zeitliche Engpässe oder Unzufriedenheiten während oder nach Ende des agilen Lernens.

Für die Rollenbesetzung ist wichtig, dass die Rollen transparent und sinnvoll besetzt sind. In einem Erprobungsfall war angestrebt worden, die fachliche Begleitung intern mit einem Mitarbeiter zu besetzen. Das Lernthema entwickelte sich jedoch so, dass dieser das benötigte Wissen gar nicht vollständig abdecken konnte, weswegen die Rolle um eine externe fachliche Begleiterin ergänzt wurde. Außerdem ist wichtig, dass alle Rollenträger die Aufgaben und Grenzen ihrer eigenen Rolle kennen. Auf diese Aufgaben und den Einfluss der einzelnen Rollenträger auf die Qualität im agilen Lernen wird weiter unten detaillierter eingegangen.

Die Evaluationsergebnisse zeigen darüber hinaus, dass die Qualität der Lernaufgaben eine besonders große Rolle für das Gelingen im agilen Lernen spielt. Die Lernaufgaben müssen das Lernthema und die Lernziele vollständig abdecken, dieses sinnvoll portionieren und gleichzeitig ermöglichen, dass die Lernenden durch das Machen eigener Erfahrungen Handlungskompetenzen aufbauen und dass sie selbstgesteuert lernen. Eine Vielzahl an Kriterien ist ausschlaggebend, wenn es darum geht, gute Lernaufgaben zu erarbeiten.

Die Lernaufgaben im agilen Lernen müssen ...

- Kontextbezug haben, d. h. sie beziehen sich auf die wirklichen Anwendungssituationen aus dem Arbeitsalltag der Lernenden,

- primäre und sekundäre Lernziele des Lernthemas abbilden,

- zur Wissensform und zur benötigten Kompetenzstufe passen,

- einer didaktischen Logik folgen,

- ganzheitliche Handlungen (planen, handeln, reflektieren) beinhalten,

- messbare und verbindliche Akzeptanzkriterien zur Selbstkontrolle haben,

- gut verständlich und zielgruppengerecht formuliert sein,

- Teamlernen fördern, d. h. sie sind nicht ausschließlich auf die Bearbeitung durch Einzelne ausgelegt,

- vom fachlichen Begleiter mit Unterstützung durch den methodischen Begleiter erstellt werden. 


\section{Durchführungsphase}

Die gesamte Lernorganisation muss sinnvoll umgesetzt sein. Das bedeutet, dass das Aufsetzen eines agilen Lernens (siehe Kap. 11) einer angemessenen Vorbereitung bedarf. Hierzu zählt neben der sinnvollen Rollenbesetzung auch ein Zeitplan (Zeitpunkt, Etappenlänge, etc.), der zum Arbeitsablauf und zu den Rahmenbedingungen der Lernteams passt.

Als besonders wertvoll bezeichnen Teilnehmer (vor allem Lernteams) das Review. Dieses Element hat - verglichen mit der Planung und der Retrospektive - einen besonders hohen Einfluss auf den Nutzen für die Lernenden wie auch für das Unternehmen. Ein wichtiger Faktor ist dabei die Feedbackqualität im Review. Fachliche Begleiter sollten Feedback auf der Aufgabenebene, d. h. konkret entlang der definierten Akzeptanzkriterien geben und anerkennen, dass der Dialog im Review Teil des Lernprozesses ist.

Die Evaluationsergebnisse zeigen außerdem, dass ein gutes Aufwand-NutzenVerhältnis aller einzelnen Meetings, d. h. Planung, Review und Retrospektive, wichtig ist. Bei gut formulierten Lernaufgaben und einem eingespielten Lernteam kann die Planung sehr kurz ausfallen, solange das Meeting seine Funktion erfüllt. Ein Review kann als ,zu langgezogen" erlebt werden, wenn Inhalte wiederholt besprochen werden oder thematisch abgeschweift wird. Gleichzeitig schätzen die Lernteams die fachliche Tiefe im Review und merken an, dass ein zu kurzes Review dem Lernprozess schadet. Die Retrospektive sollte ebenso wie die Planung bedarfsorientiert und situationsbezogen durchgeführt werden, um ein positives Aufwand-Nutzen-Verhältnis zu erzielen.

Auch das Verhältnis von Struktur und Selbststeuerung, das besonders kennzeichnend für das agile Lernen ist, sollte zum Lernteam und zum Lernthema passen. Der gesamte Ablauf folgt einer klaren Struktur - gleichzeitig hat das Lernteam vor allem während der Lernetappe viele Freiheitsgrade. Hat ein Team wenig Vorwissen in einem Lernthema und ist erstmalig für das agile Lernen zusammengestellt worden, kann eine engere Begleitung (auch während der Lernetappe) durch die fachlichen und/oder methodischen Begleiter sinnvoll und hilfreich sein. Auf der anderen Seite konnten Lernteams, die bereits Vorerfahrungen mit dem agilen Lernen und/oder dem Lerninhalt hatten, während der Lernetappe gänzlich auf die Begleiter verzichten.

Das agile Lernen ist besonders ergiebig, wenn die Potenziale seiner iterativen Struktur genutzt werden: Dazu zählen das Lernen aus Fehlern und die Anpassungen über die Etappen hinweg. Das bedeutet bspw., dass Ergebnisse aus der Retrospektive in Maßnahmen oder Anpassungen für die nächste Etappe münden. Die Umsetzung dieser Anpassungen hat der methodische Begleiter im Blick. Er reflektiert diese mit dem Lernteam in der nächsten Retrospektive usw.

Schließlich sind auch die Tools und Instrumente, die das Lernen und Arbeiten unterstützen, sinnvoll zu wählen. Sie müssen gut zur Durchführungsform des agilen Lernens passen (online vs. präsent und Vollzeit vs. arbeitsintegriert), Zugang zu den Lernaufgaben und weiteren erforderlichen Dokumenten gewährleisten und möglichst an bestehende und dem Team bekannte Tools und Plattformen ankoppeln. 


\section{Nachbereitungsphase}

Jedes agile Lernen sollte nachbereitet und nach Ende der Durchführung hinsichtlich seiner qualitativen Umsetzung kritisch reflektiert werden. War die Lernorganisation mit Blick auf Etappenlänge, Durchführungsform und eingesetzte Tools stimmig? Eignete sich das Thema wirklich für agiles Lernen und brachten die Rollenträger alle notwendigen Voraussetzungen mit? Wie zufrieden waren die Teilnehmer? Neben diesen Fragen geht es ggf. auch darum, die Inhalte und Formulierungen der Lernaufgaben nachzuschärfen oder neue Inhalte zu ergänzen. Vor allem ist aber zu prüfen, ob das Lernziel und alle relevanten Teilziele erreicht wurden.

\section{Rolle der methodischen Begleitung}

Die meisten Ansprüche werden an die Rolle des methodischen Begleiters gestellt. Für eine hohe Qualität ist die Haltung dieses Begleiters entscheidend. Die Person sollte wertschätzend kommunizieren, vermittelnd, lösungsorientiert und stärkenorientiert handeln. Sie muss die Selbststeuerung der Lernenden zulassen und fördern. Der methodische Begleiter sollte weder überfürsorglich noch überkritisch agieren und die jeweils angemessene Balance aus Aktivität und beobachtender Distanz finden.

Besonders wichtig ist eine ausgeprägte Situationskompetenz des methodischen Begleiters. Um ein gutes Aufwand-Nutzen-Verhältnis in den einzelnen Meetings zu erzielen und um die oben angesprochenen team- und themenbezogenen Anpassungen vornehmen zu können, muss der methodische Begleiter Situationen gut einschätzen, flexibel reagieren können und den Gestaltungsrahmen, den das Rahmenkonzept des agilen Lernens im Unternehmen lässt, ausschöpfen. Agiles Lernen wurde von den Teilnehmenden besonders gut bewertet, wenn der Begleiter diese Situationskompetenz zeigte und nicht nur ,starr und rigide die einzelnen Meetings durchmoderierte“. Eine Voraussetzung dafür ist ein tiefes Verständnis der jeweiligen Funktion von Planung, Review und Retrospektive. Zur Situationskompetenz gehört auch, dass der Begleiter Störungen und Besonderheiten in angemessener Weise thematisiert, ohne die Selbststeuerung der Lernenden einzuschränken.

Wichtig ist darüber hinaus die grundsätzliche Erreichbarkeit - auch während der Lernetappen. Das Lernteam sollte bei Schwierigkeiten im Lernprozess oder anderen Unsicherheiten auf den methodischen Begleiter zugehen können.

Der methodische Begleiter sollte außerdem sicherstellen, dass alle Funktionsträger (Auftraggeber, fachlicher Begleiter und Teammitglieder) ihre Rolle und die damit verbundenen Aufgaben konzeptgetreu umsetzen. Alle diese Funktionsträger sind im Prinzip ,austauschbar“ - sie variieren von Umsetzung zu Umsetzung und haben im Zweifelsfall keine Vorerfahrung mit dem agilen Lernen im Unternehmen. Hingegen liegt die methodische Kompetenz für das agile Lernen beim methodischen Begleiter, der in der Regel unterschiedliche Umsetzungen begleitet. Dementsprechend gehört es zu seinen Aufgaben, andere Personen in ihre Rollen einzuführen und im Prozess bei Bedarf zu intervenieren. So könnte beispielsweise ein fachlicher Begleiter seine Aufgabe im Verlauf des agilen Lernens (unbeabsichtigt oder unbewusst) verlassen, indem er den Lernenden Vorträge hält und damit die Aktivität und Selbststeuerung der Lernenden untergräbt. In diesem Szenario 
obliegt es dem methodischen Begleiter, die Situation einzuschätzen und auf eine konzeptgetreue Umsetzung der Rollen zu achten.

\section{Rolle Teammitglied}

Die Ergebnisse zeigen, dass die Teammitglieder grundsätzlich dazu bereit sein müssen, die geforderte Selbststeuerung anzunehmen. Dabei kann es sein, dass einzelne Teammitglieder andere mitziehen oder dass Einzelne die Selbststeuerung des gesamten Lernteams besonders stark umsetzen. Dennoch ist die grundsätzliche Bereitschaft zur Selbststeuerung bei jedem einzelnen Teammitglied eine wichtige Bedingung für das Gelingen des agilen Lernens. Außerdem müssen die Lerner den Lernprozess als Team aktiv gestalten. Es gibt zwar fachliche und methodische Unterstützung, der Kern des agilen Lernens besteht aber im Lernen durch eigene Erfahrungen, so dass das Team aktiv arbeiten, diskutieren und Ergebnisse festhalten muss. Entsprechend wichtig ist eine positive Haltung zu Kooperation. Jedes Teammitglied sollte bereit sein, Wissen zu teilen und andere Teammitglieder zu unterstützen - auch wenn diese möglicherweise geringere Vorkenntnisse zu einem Lernthema haben. Eine konkurrenzgetriebene Grundhaltung schadet dem Lernprozess und der Atmosphäre im Team.

\section{Rolle Auftraggeber}

Für eine hohe Qualität im agilen Lernen ist entscheidend, dass der Auftraggeber eindeutig bestimmt ist und seine Ziele und Erwartungen klar benennt. Diese Aspekte müssen gut ineinandergreifen - ansonsten können im späteren Verlauf Unstimmigkeiten zwischen Anspruch, zeitlichem Invest und Zufriedenheit der Beteiligten entstehen. Es zeigte sich auBerdem, dass der Auftraggeber auf die Zielerreichung für das gesamte Vorhaben achten und diese nachhalten sollte. Damit ist nicht gemeint, dass der Fortschritt regelmäßig kontrolliert wird. Es geht vielmehr darum, dass der erfolgreiche und vollständige Abschluss des Lernvorhabens im „,natürlichen Interesse“ des Auftraggebers liegt. Andernfalls kann sich aus der iterativen Struktur des agilen Lernens sogar eine Gefahr ergeben: Die Motivation des Teams könnte über die Zeit sinken, wenn es merkt, dass Relevanz und/oder Priorität des Themas für den Auftraggeber abnimmt oder nicht vorhanden ist. In der praktischen Umsetzung sollte der Auftraggeber seinen Bedarf und sein Interesse also transparent erläutern und sich zumindest nach der Durchführung des agilen Lernens Zeit dafür nehmen, über die Ergebnisse informiert zu werden.

\section{Rolle der fachlichen Begleitung}

Zunächst ist wichtig, dass die fachlichen Begleiter das benötigte Wissen und die benötigten Kompetenzen abdecken. Dabei kann es vorkommen, dass bestimmte Personen nur punktuell (d. h. für einzelne Lernaufgaben) als Fachexperten fungieren und nicht in alle Etappen des agilen Lernens eingebunden sind. Das benötigte Wissen muss aber - intern oder extern - vorhanden sein und es muss geklärt sein, wessen Ressourcen für die Rolle des fachlichen Begleiters eingesetzt werden. Es hat sich außerdem gezeigt, dass auch bei den fachlichen Begleitern Aspekte der Haltung Einfluss auf die Qualität im agilen Lernen 
haben. Sie müssen ebenfalls die Selbststeuerung der Lernenden unterstützen, Teamlernen zulassen und anerkennen, dass aus Fehlern gelernt werden kann.

Für eine besonders hohe Qualität im agilen Lernen sind die fachlichen Begleiter in der Lage, bedarfsbezogen abzuschätzen, welche Art von Unterstützung den Lernprozess des Teams zielgenau fördert. Die Evaluationsergebnisse zeigen, dass fachliche Begleiter, die diese Rolle im agilen Lernen schon mehrfach eingenommen hatten, bei einer Rückfrage des Teams zunächst abwägten, welches Vorgehen für die Lernenden am hilfreichsten sein würde: Je nach Ausgangslage stellten sie für einen optimalen Lernprozess mehrere Schritte eines Lösungswegs dar, erklärten die gesamte Lösung oder aber gaben nur einen Tipp zur Lösung ohne jeglichen Lösungsschritt.

\subsection{Ein Qualitätssicherungskonzept für das agile Lernen im Unternehmen}

Welche Maßnahmen und Empfehlungen lassen sich daraus ableiten, um eine hohe Qualität bei den Umsetzungen des agilen Lernens in Unternehmen zu erzielen? Diese Frage wurde im Vorhaben inMEDIASres ausgehend von den Evaluationsergebnissen und Erkenntnissen zur Qualitätssicherung für das agile Sprintlernen beantwortet. Die folgende Übersicht fasst diese Anforderungen zusammen:

- Die Vorbereitung des agilen Lernens im Unternehmen folgt den Schritten zur Vorbereitung und zum guten Abschluss eines agilen Lernprojekts. Die systematische Gestaltung wird laufend gepflegt (Kap. 11).

- Es gibt einen qualifizierten Process Owner für agiles Lernen im Unternehmen, der selbst auch qualifizierter methodischer Begleiter ist.

- Jedes agile Lernen wird durch einen qualifizierten methodischen Begleiter verantwortet und begleitet.

- Tools, Checklisten und Leitfäden für qualitativ hochwertiges agiles Lernen im Unternehmen sind verfügbar und werden laufend aktualisiert.

- Es gibt einen Prozess zur systematischen Reflexion des Handelns der methodischen und der fachlichen Begleiter, z. B. einen regelmäßigen direkten und strukturierten Austausch der Begleiter untereinander und mit den Teams.

- Für jedes Lernangebot sind klare Kommunikationswege für zielgenauen und bedarfsbezogenen Austausch zwischen den beteiligten Funktionsträgern angelegt.

- Für jedes agile Lernen wird eine Evaluation aufgesetzt und durchgeführt. Mindestens zu betrachten sind Zielerreichung und Akzeptanz der Lernform.

- Erkenntnisse aus der Evaluation münden in eine Anpassung des Angebots oder in andere Maßnahmen. 


\subsection{Fazit}

Alles in allem zeigt sich, dass agiles Lernen im Unternehmen mit all seinen Potenzialen bei weitem nicht voraussetzungsfrei ist. $\mathrm{Zu}$ einer wirklich guten und qualitativ hochwertigen Umsetzung gehört eine passende Qualifizierung und letztlich auch Erfahrung, insbesondere was die Rolle des methodischen Begleiters betrifft. Agiles Lernen hat sehr viele strukturgebende Elemente - wobei die Herausforderungen häufig im Detail liegen. Neben recht hohen Ansprüchen an die didaktische Aufbereitung (insbesondere der Lernaufgaben) sind vor allem ,weiche Faktoren“ und Kompetenzen wie die Haltung der Beteiligten und die Situationskompetenz des methodischen Begleiters entscheidend für eine hohe Qualität. Wird agiles Lernen ohne jegliche Vorerfahrung und ohne Beachtung dieser weichen Faktoren umgesetzt, besteht die Gefahr, diese Lernform zu ,verbrennen“, weil die Beteiligten den Sinn nicht erkennen können, ein schlechtes Aufwand-Nutzen-Verhältnis erleben oder wenig Nachhaltigkeit im Lernen erleben.

Unternehmen, die das agile Lernen im Unternehmen intensiv nutzen oder bei sich verankern wollen, sollten die Maßnahmen des Qualitätssicherungskonzepts strukturiert umsetzen, um den größtmöglichen Nutzen mit dem agilen Lernen zu erzielen: jederzeit schlank bedarfsgerecht jene Kompetenzen aufbauen zu können, die letztlich nur durch Erfahrungslernen zu erwerben sind.

Open Access Dieses Kapitel wird unter der Creative Commons Namensnennung - Weitergabe unter gleichen Bedingungen 4.0 International Lizenz (http://creativecommons.org/licenses/ by-sa/4.0/deed.de) veröffentlicht, welche die Nutzung, Vervielfältigung, Bearbeitung, Verbreitung und Wiedergabe in jeglichem Medium und Format erlaubt, sofern Sie den/die ursprünglichen $\mathrm{Au}-$ tor(en) und die Quelle ordnungsgemäß nennen, einen Link zur Creative Commons Lizenz beifügen und angeben, ob Änderungen vorgenommen wurden. Wenn Sie das Buch oder Teile daraus remixen, verändern oder anderweitig direkt darauf aufbauen, dürfen Sie Ihre Beiträge nur unter derselben Lizenz wie das Original verbreiten.

Die in diesem Kapitel enthaltenen Bilder und sonstiges Drittmaterial unterliegen ebenfalls der genannten Creative Commons Lizenz, sofern sich aus der Abbildungslegende nichts anderes ergibt. Sofern das betreffende Material nicht unter der genannten Creative Commons Lizenz steht und die betreffende Handlung nicht nach gesetzlichen Vorschriften erlaubt ist, ist für die oben aufgeführten Weiterverwendungen des Materials die Einwilligung des jeweiligen Rechteinhabers einzuholen.

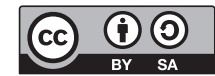

